\title{
A novel concept for actinic EUV mask review tool using a scanning lensless imaging method at the Swiss Light Source (Withdrawal Notice)
}

Sangsul Lee, Manuel Guizar-Sicairos, Yasin Ekinci

Sangsul Lee, Manuel Guizar-Sicairos, Yasin Ekinci, "A novel concept for actinic EUV mask review tool using a scanning lensless imaging method at the Swiss Light Source (Withdrawal Notice)," Proc. SPIE 9048, Extreme Ultraviolet (EUV) Lithography V, 904811 (17 April 2014); doi: $10.1117 / 12.2046226$ 


\title{
A novel concept for actinic EUV mask review tool using a scanning lensless imaging method at the Swiss Light Source (Withdrawal Notice)
}

\author{
Proc. SPIE 9048, 904811 (2014) \\ Online Publication Date: 17 April 2014 \\ Withdrawn from Publication: 25 May 2016
}

Conference Date: 24-27 February 2014

Conference Location: San Jose, California, United States

Conference Title: Extreme Ultraviolet (EUV) Lithography V

Conference Chairs: Obert R. Wood II, Eric M. Panning

Sangsul Lee, Manuel Guizar-Sicairos, Yasin Ekinci

Paul Scherrer Institut (Switzerland)

Publisher's Note: This paper, originally published on April 17, 2014, was withdrawn at the author's request on May 25, 2016. (c) (2014) COPYRIGHT Society of Photo-Optical Instrumentation Engineers (SPIE). 